\title{
EVALUATION OF BONE-TO-IMPLANT CONTACT AND BONE DENSITY ADJACENT TO TITANIUM IMPLANTS USING A STEREOLOGICAL TECHNIQUE ON GROUND SECTIONS
}

\author{
Dimitra BALATSOUKA ${ }^{1}$, KLAUs GOTFREDSEN ${ }^{1}$ AND HANS JøRGEN G GUNDERSEN ${ }^{2}$ \\ ${ }^{1}$ Department of Prosthetic Dentistry, School of Dentistry, Health Science Faculty, University of Copenhagen, \\ Nørre Allé 20, 2200 Copenhagen N, Denmark; ${ }^{2}$ Stereological Research Laboratory, Aarhus University \\ Hospital, DK-8000 Aarhus C, Denmark. \\ e-mail: dbalatsouka@yahoo.com, klg@odont.ku.dk, stereohj@post.tele.dk \\ (Accepted March 7, 2006)
}

\begin{abstract}
When bone implants have to be examined in situ ground sections are required. Histomorphometric measurements are usually performed on two-dimensional sections, causing biased results when they are wrongly extrapolated to 3D without any knowledge of stereology. Unbiased results can only be obtained using stereological principles. The aim of the study was to describe an unbiased design for evaluating boneto-implant contact $(B I C)$ and peri-implant bone density $(B D-i)$ in three-dimensions. The unbiased design was based on a fixed axis vertical random sampling technique. Three bone-implant blocks were collected from 3 rabbits. Four sections were obtained from each animal using a fixed axis vertical random sampling technique. The $B I C$ was estimated by creating a stereological method based on a systematic test line set. The $B D-i$ was estimated using a design based on a systematic point set. The efficiency of the systematic sampling was evaluated for each sampling level: Coefficient of error $C E\left(\bar{w}_{i}\right)$ for the systematic test line set, $C E_{S y s t}$ between the 4 rotated sections, $C E_{\text {Noise }}$ for the reproducibility. These variances were compared to the biological variation $(C V)$ between animals. The mean $C E\left(\bar{w}_{i}\right)$ was 5 to $6 \%$; the $C E_{\text {Syst }}$ was $7.8 \%$ for the $B I C$ estimates and $5.7 \%$ for the $B D-i$ estimates; the $C E_{\text {Noise }}$ was $5.8 \%$ for the $B I C$ and $7.7 \%$ for the $B D-i$. The $C V$ was $19 \%$ for the $B I C$ estimates and $24 \%$ for the $B D-i$ estimates. These results demonstrated that the stereological technique used in the present study was a very efficient method to obtain unbiased estimates of $B I C$ and $B D-i$ in $3 \mathrm{D}$ on $2 \mathrm{D}$ implant-bone sections.
\end{abstract}

Keywords: efficiency, histomorphometric measurements, stereology, systematic sampling, vertical sections.

\section{INTRODUCTION}

Histological implant studies frequently use the definition of osseointegration as: "A direct, on the light microscopical level, contact between living bone and implant" (Albrektsson et al., 1981). Although this definition does not state the proportion of bone in contact with the implant surface, the fraction of surface areas of mineralized bone in contact with the implant surface $(B I C)$, is one of the most used variables in histomorphometric analysis of implants (Albrektsson, 1988; Sennerby et al., 1991). Another most frequently used variable in histological implant studies is the peri-implant bone density $(B D-i)$, which is the volume fraction of mineralized bone in a periimplant area (Johansson, 1991; Ivanoff et al., 1997; Gotfredsen et al., 2001).

The $B I C$ and $B D-i$ measurements have traditionally been made on a few central ground sections cut in a fixed orientation and parallel to the long axis of the implants (Gotfredsen et al., 1993; Ivanoff et al., 1997; Gotfredsen et al., 2001). In most studies a bucco-lingual direction has been selected (Rasmusson et al., 2001; Fini et al., 2002; Kolonidis et al., 2003). In other studies a mesio-distal direction has been chosen (Gotfredsen et al., 1993; Gotfredsen et al., 2001; Romanos et al., 2002) and examples of both mesio-distal and bucco-lingual direction are also present (Gotfredsen et al., 1991; Schou et al., 2003).

Ground sections with the implant in situ are normally relatively thick sections $(20-40 \mu \mathrm{m})$, which means that only a few sections can be obtained from a bone-implant block (Gotfredsen et al., 1989). Histomorphometric measurements on 2-dimensional ground sections may therefore be difficult to extrapolate to 3D. Although these sampling methods have been performed with a high precision the results 
may have been biased, because the sampling methods have not fulfilled stereological principles. Stereology is a precise tool for obtaining quantitative information about 3-dimensional structures, based on observation made on 2-dimensional sections (Gundersen et al., 1988). Bone is an inhomogeneous and anisotropic structure and 2-dimensional histomorphometric methods should therefore include stereological principles to provide unbiased estimates for the 3dimensional structure (Baddeley et al., 1986; Gundersen et al., 1988). The traditional methods for describing the $B I C$ and the $B D-i$ do not respect these principles of uniform sampling and biased results may occur, which result in erroneous conclusions.

Histomorphometric parameters like $B I C$ and $B D-i$ are defined in terms of the implant, which is an artificial structure of precisely known, constant dimensions. In order to obtain efficient and unbiased estimates of $B I C$ and $B D-i$, it is important to plan the sampling method before processing and sectioning the specimens. Practical efficiency means to obtain an estimate with a low variability after spending a moderate amount of time and unbiased estimation means estimation without systematic deviation from the true value (Gundersen et al., 1988). Generally, to have unbiased estimates, sampling must be uniformly random, and to increase efficiency systematic sampling designs are recommended.

Using the "vertical sectioning" technique (Baddeley et al., 1986; Vesterby et al., 1987) these requirements have been respected (Overgaard, 2000; Overgaard et al., 2000; Rahbek, 2002). However, when BIC and $B D-i$ measurements have to be performed on ground sections a "shadow effect" or underprojection occur on sections, where the cut is not close to the center axis (Fig. 1). Therefore, to reduce problems and the potential bias from the "shadow effect" the most central sections have usually been selected for $B I C$ and BD-i measurements (Overgaard et al., 1999; Gotfredsen et al., 2000; Kolonidis et al., 2003). This means that only a few sections are available for the $B I C$ and $B D-i$ measurements, and the sampling is not uniformly random.

The above problems may, however, be solved using a fixed axis vertical random sampling technique and the "revolving door principle" (Cruz-Orive 1987; Cruz-Orive and Roberts 1993; Jensen and Gundersen 1993), in a design described below. This method offers a unique possibility to obtain robust and unbiased estimates of $B I C$ and $B D-i$, respectively, in implant-bone specimens.

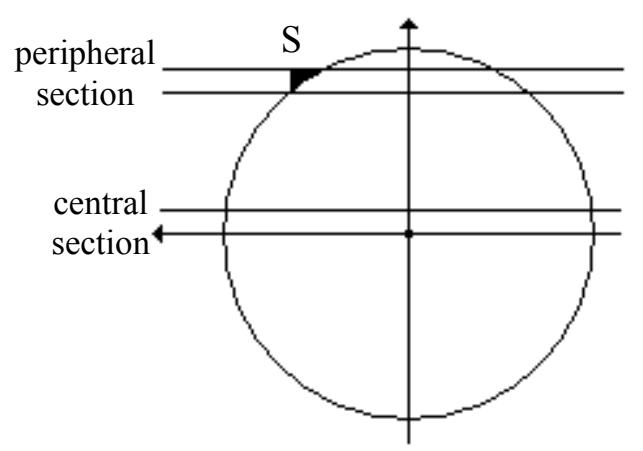

Fig. 1. The shadow effect. When a cut is made through the center axis of the implant (central section) the shadow effect (S) from the cervical curve is very small. When a cut is made in the periphery of the implant (peripheral section) the shadow effect is increased.

The aim of this study was to describe a stereological design for estimating bone-to-implant contact $(B I C)$ and peri-implant bone density $(B D-i)$ on ground sections in three-dimensions. Note that ordinary vertical, uniformly random (VUR) sections must have uniformly random position. Coaxial sections, with a fixed (arbitrary) position of the axis, cannot in general provide unbiased estimates of surface area, nor will ordinary point counting provide unbiased estimates of volume.

\section{MATERIALS AND METHODS}

\section{EXPERIMENTAL ANIMALS AND IMPLANT PLACEMENT}

Three bone-implant specimens were collected from 3 pilot female white New Zealand rabbits (SSc: $\mathrm{CPH}$, Statens Serum Institute), weighing 3 to $4 \mathrm{~kg}$ and with an age of 9 to 12 months. Experimental screw-formed implants made of commercially pure titanium and with a $\mathrm{TiO}_{2}$-blasted surface (Astra Tech, Mölndal, Sweden) were placed in the proximal part of the tibia. The implants had an outer diameter of 3.5 $\mathrm{mm}$ and were $6 \mathrm{~mm}$ in length, whereby only one cortical layer was engaged. After placing the implants, the fascia-periosteal parts of the flaps were closed with single resorbable sutures (Vicryl ${ }^{\circledR} 4-0$, Ethicon, Norderstedt, Germany), while non-resorbable sutures (Prolene $^{\circledR}$ 5-0, Ethicon) were used for the skin. Postoperatively, all animals received antibiotic treatment for 3 days $(1.5 \mathrm{ml} /$ day Streptocillin, Boehringer, Ingelheim, Copenhagen, Denmark A/S) and analgetic treatment for 1 day $(0.5 \mathrm{ml} /$ day Temgesic $0.3 \mathrm{mg} / \mathrm{ml}$, Schering-Plough Europe, Brussels, 
Belgium) given as single intramuscular injections. The animals were sacrificed with an overdose of sodium pentobarbital (Pentobarbital, $200 \mathrm{mg} / \mathrm{ml}$; The Royal Veterinary and Agricultural Pharmacy, Frederiksberg, Denmark; $2 \mathrm{ml}$ per animal). The National Ethical Committee on Animal Experiments approved the study protocol (J. nr. 2001/561-421).

\section{HISTOLOGY AND STEREOLOGY}

The bone-implant specimens were osteotomised and immersed in 10\% formalin. The specimens were dehydrated in graded series of ethanol and embedded in methyl methacrylate-based resin (Technovit 7200, Kulzer, Freidrichsdorf, Germany).

Each embedded bone-implant block was coded and rotated randomly around the vertical axis of the implant (Fig. 2a). The bone-implant blocks were then sectioned through the implant centre into four equal parts parallel to the long axis of the implant, which is a fixed vertical axis (Fig. 2a). This was accomplished using an X-ray-guided technique (Fig. 3a,b). When the vertical axis was identified for the rotated boneimplant block, the block was split into two halves and both of the halves were furthermore divided into two equal quarters using a diamond saw with a trail-width of $0.1 \mathrm{~mm}$ (Fig. 2a). Thereby 4 identical and separate specimens $A, B, C$ and $D$ were obtained (Fig. 2b). Each quarter was placed in embedding molds in such a way that the surface for sectioning was placed towards the bottom of the mold. Four central boneimplant sections of each implant without "shadow effect" were then obtained. The 4 vertical sections were not taken at random and independently, but the first one was taken at random and the other three at right angles i.e. "revolving-door" principle. The sections were ground to a thickness of approximately $25 \mu \mathrm{m}$ and stained with toluidin blue.

Stereological quantitation was performed in a darkened room using a Leica DMLB microscope (Leica Microscopy Systems Ltd, Heerbrugg, Switzerland) with a projection arm transmitting the section image perpendicularly to the table next to the microscope.
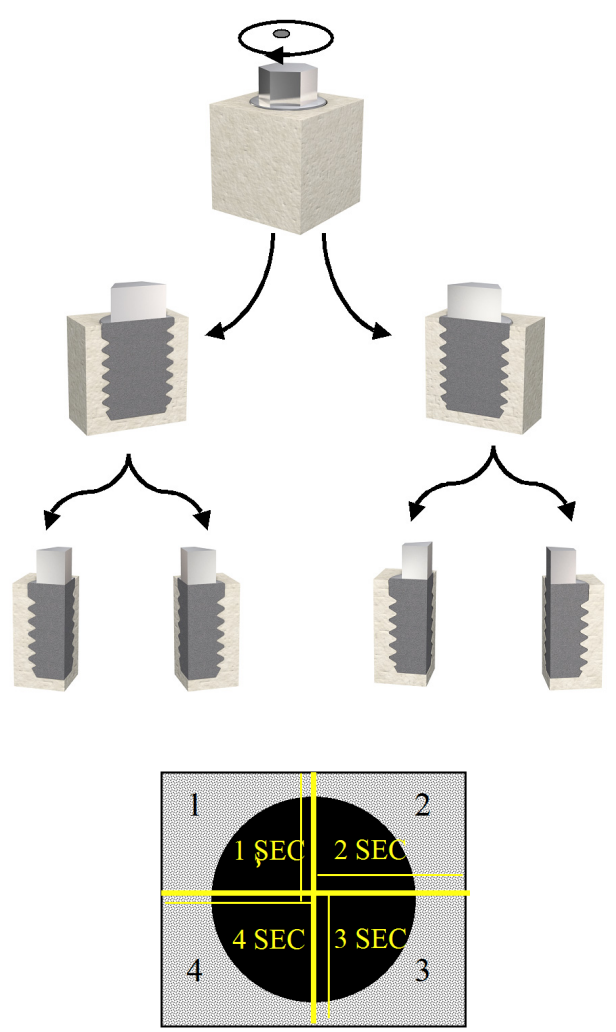

Fig. 2. A) The preparation procedure. The boneimplant block was randomly rotated and cut through the middle of the vertical axis. Each of the two half was re-embedded and cut through the middle into two quarters, which also was re-embedded before sections were taken according to $b$. B) After rotation the boneimplant block was split into four specimens and four central bone-implant sections were obtained.
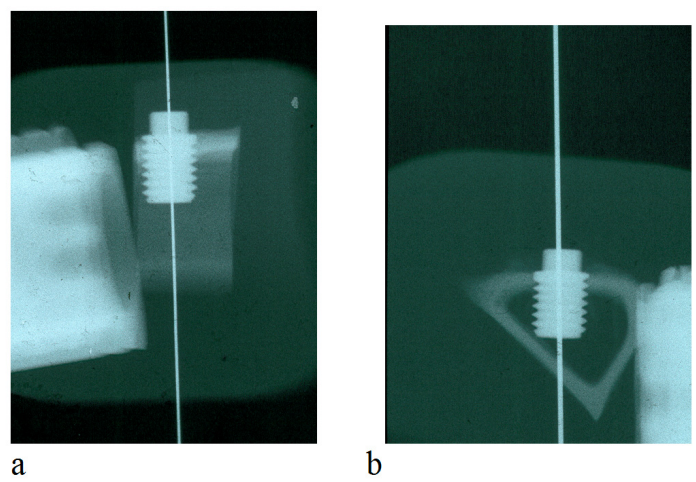

Fig. 3. Division in a frontal (a) and a median (sagital) plane (b) of the bone-implant block using an $X$-ray guided technique. 


\section{A STEREOLOGICAL METHOD FOR ESTIMATING ABSOLUTE SURFACE AREAS AND BIC ON COAXIAL SECTIONS}

\section{Design}

Coaxial sections do normally not allow surface estimation of three-dimensional structures. However, the dental implant is a precisely known artefact with an unusual degree of regularity and symmetry making it possible not only to estimate volumes, as the $B D-i$ around it, but also to estimate surfaces, as the $B I C$, in a very efficient way.

The surface areas of the implant and of the BIC were evaluated according to a new estimator for the surface area fraction, which at any point is:

1. at an observable and varying distance $r$ from the axis of rotation, which in turn is identical to the fixed vertical axis of the stereological design (cf. Figs. 4 and 5).

2. everywhere perpendicular to the plane of the coaxial section, but has a varying and observable angle $\alpha$ with respect to the vertical axis, as shown in Fig. 5.
The amount of surface around the implant is at any point proportional with the distance $r$ from the axis and with the $\cos \alpha$, where $\alpha$ is the angle between the surface profile and the vertical axis.

When the implant-bone surface is intersected by horizontal test lines, perpendicular to and randomly displaced along the axis of the implant (Fig. 4), each intersection point $\left(I_{i}\right)$ represents a known amount of 3-dimensional surface area $s_{i}$ :

$$
s_{i}=d \cdot \frac{\pi}{2} \cdot \mathrm{r}_{\mathrm{i}} \frac{1}{\sin \alpha_{i}}
$$

where $d$ is the constant distance between test lines and $\pi / 2\left(90^{\circ}\right)$ is the rotational sampling period (Figs. 4 and 5). The factor $r_{i}$ signifies that the amount of surface is directly proportional with the distance from the axis and the factor $1 / \sin \alpha_{i}$ (the angle between the surface profile and the test line) compensates for the fact that the test lines only detect the projection of the surface onto the axis. Suffix $i$ indicates that the variable depends on the position. Note that the equation is only valid when the fixed axis of the coaxial section coincides with that of the implant.

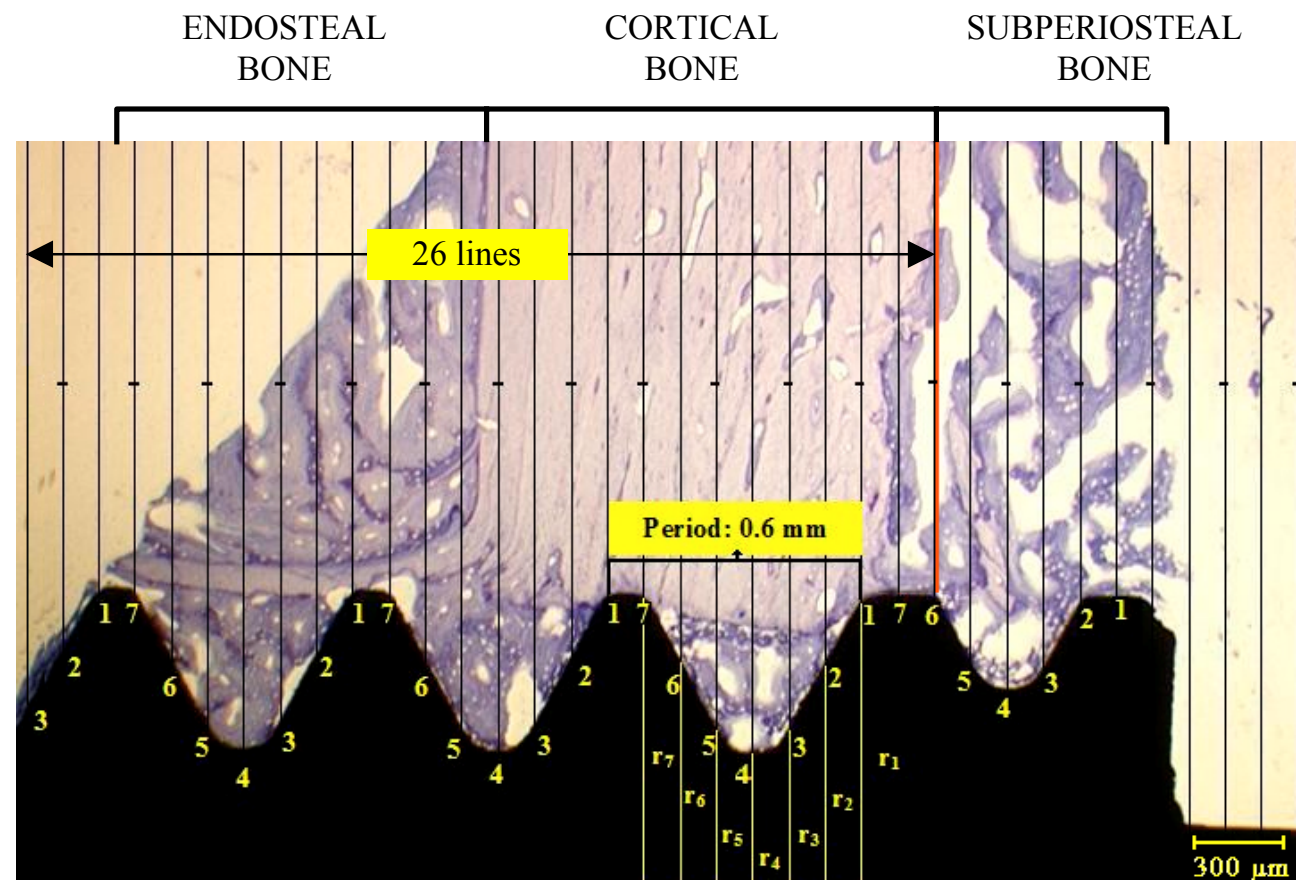

Fig. 4. A systematic test line orientated perpendicularly to the axis of the implant placed over one section. The axis of the implant is the lower edge of the image. One bone thread (period) was divided into 7 intervals, each represented by a numbered line $(1,2,3,4,5,6,7)$. For each line the distance $r_{i}$ from the implant surface to the axis of the implant was observable. For each section, intersections with 26 lines were counted from the upper level of the cortical bone to half of the implant's length. 


\section{The test system and its construction}

For creating a systematic test line set appropriate for estimating surfaces in these coaxial sections, the distance from the top of the thread to the top of the next one (the thread period) was measured precisely. This determination was made on 12 sections, i.e., all four coaxial sections on three bone-implant specimens. The distance (period) between each implant thread was found to be $0.6 \mathrm{~mm}$ (Fig. 4). The surface estimation procedure was made much more efficient by constructing a set of numbered test lines for which $d \cdot c=$ length, where $d$ is the constant distance between test lines; $c$ was arbitrarily fixed at $c=7$. In this way, each period was divided into 7 intervals by 7 test lines, placed uniformly randomly and perpendicular to the fixed axis of the implant (Fig. 4).

For estimating the constants of the test system, each interval was furthermore arbitrarily divided into 3 subintervals (points) $j=a, b$, or $c$ and for each midpoint of the subintervals the radius $\left(r_{i j}\right)$ was measured and divided with the sinus to the angle $\left(\alpha_{i j}\right)$ (Fig. 5).

$$
w_{i j}=\frac{r_{i j}}{\sin \alpha_{i j}} .
$$

Always numbering the lines from the top of a thread, the value of $w_{i j}$ of each subinterval (points) varies very little for a given interval and treating the mean $w_{i}$ of each interval as a constant only introduces a small variation, monitored by $C E=S E M /$ mean, in the estimate, where $S E M$ is the standard error of the mean.

The averaging was over the 3 subintervals $j=a$, $b$, or $c$ in each interval:

$$
w_{i}=\frac{\sum_{j=a}^{j=c} r_{i j}}{\sum_{j=a}^{j=c} \sin \alpha_{i j}} .
$$

For estimating the $C E$ of the test line system itself, $w_{i}$ was determined in all sections for all animals, giving a mean $\bar{w}_{i}$ for each interval and the mean $C E\left(\bar{w}_{i}\right)$ was averaged over all seven intervals:

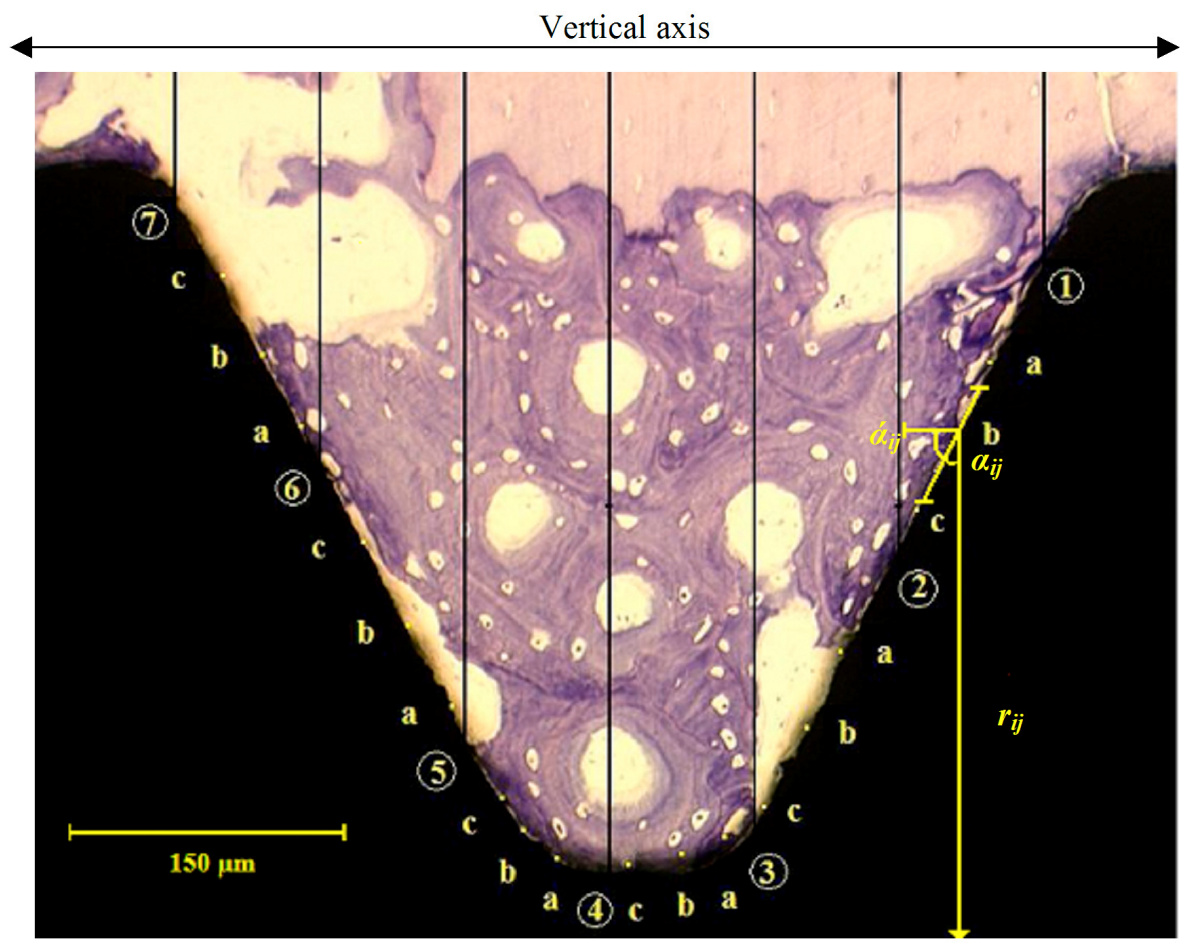

Fig. 5. Each interval was divided into 3 subintervals $(a, b, c)$ and for each mid point of the subintervals the radius $\left(r_{i j}\right)$ was measured and divided with the sinus to the angle $\left(\alpha_{i j}\right) . \alpha_{i j}$ is the angle between the surface and the test line, while $\dot{\alpha}_{i j}$ is the angle between the surface and the vertical axis of the implant; cos $\dot{\alpha}_{i j}=\sin \alpha_{i j}$. 
As shown in Table 1 , the mean $C E\left(\bar{w}_{i}\right)$ was 5 to $6 \%$, both for complete threads and for the first, incomplete thread. For all animals in the study the set of coefficients $\bar{w}_{i}$ shown in Table 1 were thus known constants for each test line, numbered from the top of a complete thread. Note the large variation of $\bar{w}_{i}$ by a factor of almost 3 as a function of the position along the thread. By using the individual coefficients (instead of their global mean) in the estimation described below, the estimates are unbiased under the (exactly fitting) stereological model of the implant surface. If the global mean is used, the estimates would be biased by e.g. a non-uniform distribution of $B I C$ along the surface of the implant.

All previous measurements were made for the complete threads of the implant. For areas on the first incomplete thread, 7 individual interval values of $\bar{w}_{i}$ were calculated separately (Fig. 6, Table 1).

Table 1. Each period (thread) was evaluated using 7 test lines. Each line was characterized by its number (starting at the top of a thread). $\bar{w}_{i}$ is the mean value of $w_{i}$, averaged first over the four sections in each animal and then over the three animals. Mean $C E\left(\bar{w}_{i}\right)$ is an average over the 7 test lines.

\begin{tabular}{ccccc}
\hline \multirow{2}{*}{ Surface area } & \multicolumn{2}{c}{ Complete threads } & \multicolumn{2}{c}{ Incomplete 1 st thread } \\
\cline { 2 - 5 } Test line & $\bar{w}_{i}$ & CE\% & $\bar{w}_{i}$ & CE\% \\
\hline 1 & 2.59 & 4.2 & 2.24 & 8.4 \\
2 & 3.69 & 5.4 & 3.32 & 5.1 \\
3 & 2.71 & 4.6 & 3.01 & 4.1 \\
4 & 1.45 & 1.5 & 1.53 & 2.8 \\
5 & 3.08 & 8.6 & 2.66 & 3.3 \\
6 & 3.95 & 2.2 & 3.76 & 6.3 \\
7 & 2.35 & 8.9 & 2.44 & 6.2 \\
\hline & mean $C E\left(\bar{w}_{i}\right)$ & 5.7 & mean $C E\left(\bar{w}_{i}\right)$ & 5.5 \\
\hline
\end{tabular}

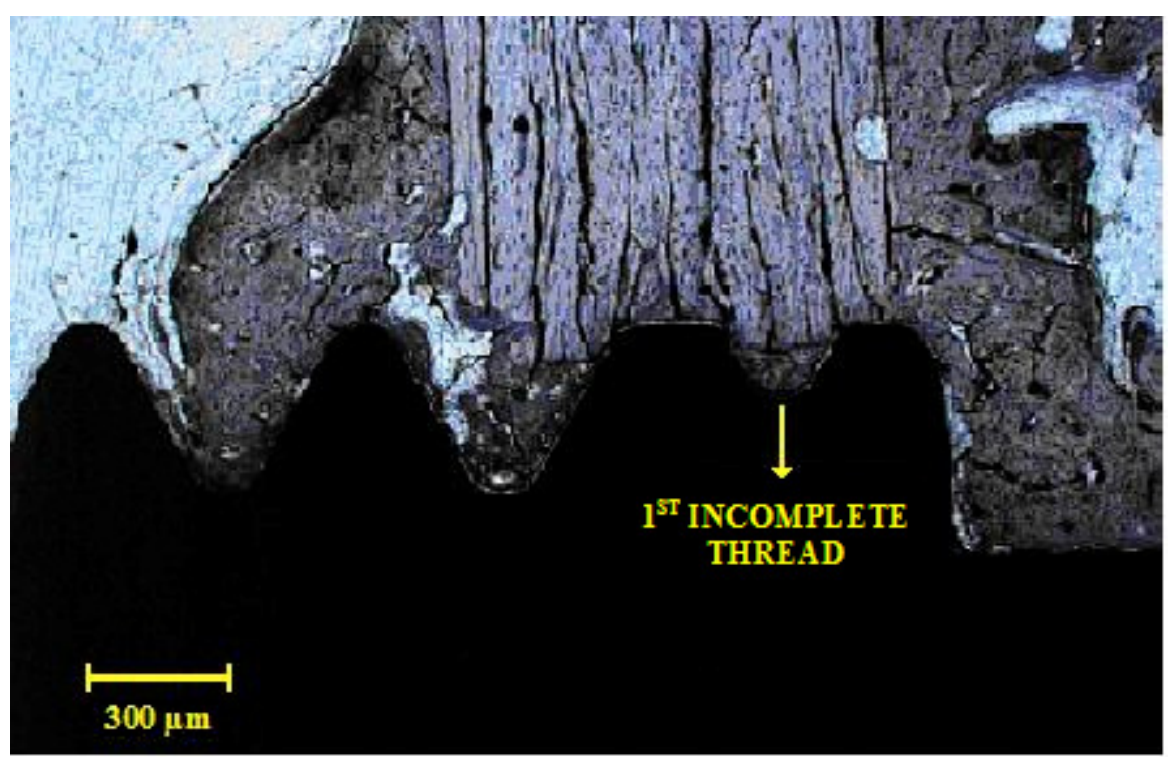

Fig. 6. The first thread was normally incomplete and individual distances and angles were calculated for this thread separately (Table 1). 


\section{BIC ESTIMATED USING A STEREOLOGICAL DESIGN ON COAXIAL SECTIONS}

The $B I C$ was determined from the upper level of the cortical bone to half of the implant's length (Fig. 4) at a final magnification of $72 \times$. The half implant length was chosen to estimate BIC because it corresponds to the three best consecutive threads of the implant. The three best consequitive threads correspond to the cortical region of the tibia of the animal. In the tibial bone bed there is no cancellous bone in the marrow cavity in the region of the implants, which means that the last half length of the implant has no bone contact because no bone is growing there. A systematic test line orientated perpendicularly to the vertical axis of the implant was placed over the projected image of the 4 "revolvingdoor" sections (Fig. 4). For each section, intersections with 26 lines were counted (Fig. 4). Presence or absence of bone-to-implant contact was registered at all intersections between test lines and implant surface. The fractional area of mineralised bone in contact with the implant $(B I C)$ was then estimated by the following equation:

$$
B I C \%=\frac{\sum\left[I_{i}(\text { BIC }) \cdot \bar{w}_{i}\right]}{\sum\left[I_{i}(\text { Total }) \cdot \bar{w}_{i}\right]} .
$$

Where $I_{i}(B I C)$ is the number of intersections with the implant surface covered by minerilised bone and $I_{i}$ (Total) is the total number of intersections with the implant surface threads. The summation is over 26 test lines per section and 4 sections per specimen. The final $C E$ of this estimator is dealt with below. The absolute surface area of $B I C$ per implant may be estimated as:

$$
S(B I C)=\frac{\pi}{2} \cdot d \cdot \sum\left[I_{i}(B I C) \cdot \overline{w_{i}}\right],
$$

\section{BD-i ESTIMATED USING A STEREOLOGICAL DESIGN ON COAXIAL SECTIONS.}

\section{Design}

In histological implant studies, the bone density $(B D-i)$ is usually defined as the fraction of mineralized bone in a peri-implant area. For threaded implants the amount of bone between the implant threads has frequently been used as the area for $B D-i$ 2D measurements (Johansson, 1991; Ivanoff et al., 1997). By obtaining coaxial sections unconditionally unbiased estimates of 3D bone density are provided and also a better delineation of the peri-implant regions because they are transversely sectioned. A new design was developed in order to obtain maximal efficiency for estimating bone volume factions.

\section{Test system}

A systematic point set was placed randomly over the bone-implant specimen but with the rows of points parallel to the vertical axis of the implant. The density of the systematic point set was chosen so as to provide 100-200 hits on 4 sections (Cruz-Orive and Weibel, 1990). Thus, 5 rows of points were counted in each bone thread (Fig. 7). Each point represented an area $\left(a_{p}\right)$, determined by the distance $u$, between 2 neighbouring points, corrected for magnification: $a_{p}=u^{2}$.

\section{Estimation procedure}

In the present study the bone density $(B D-i)$ was estimated from the upper level of the cortical bone to half of the implant's length, at a magnification of $146 \times$.

The points $(P)$ hitting the mineralized bone within the threads were counted and the distance $(r)$ from the point to the vertical axis of the implant was measured (Miranov \& Miranov 1998), (Fig. 7). The BD was determined by the following equation:

$$
B D \%=\frac{\sum_{i=1}^{i=5} P_{B i} \cdot r_{i}}{\sum_{i=1}^{i=5} P_{T i} \cdot r_{i}}
$$

where $P_{B 1}, P_{B 2}, \ldots P_{B 5}$ are the points hitting bone at distances $r_{1}, r_{2}, \ldots r_{5}$, and $P_{T 1}, P_{T 2}, \ldots P_{T 5}$ are the total number of points at distances $r_{1}, r_{2}, \ldots r_{5}$ (Fig. 7). Since the distance between the rows of points is constant and known, the distance to the axis only has to be measured for one row on one section. Note that ordinary point counting fails on coaxial sections because the amount of bone increases with the distance to the section, a fact which is taken into account with the factor $r_{i}$ in the above equation. 


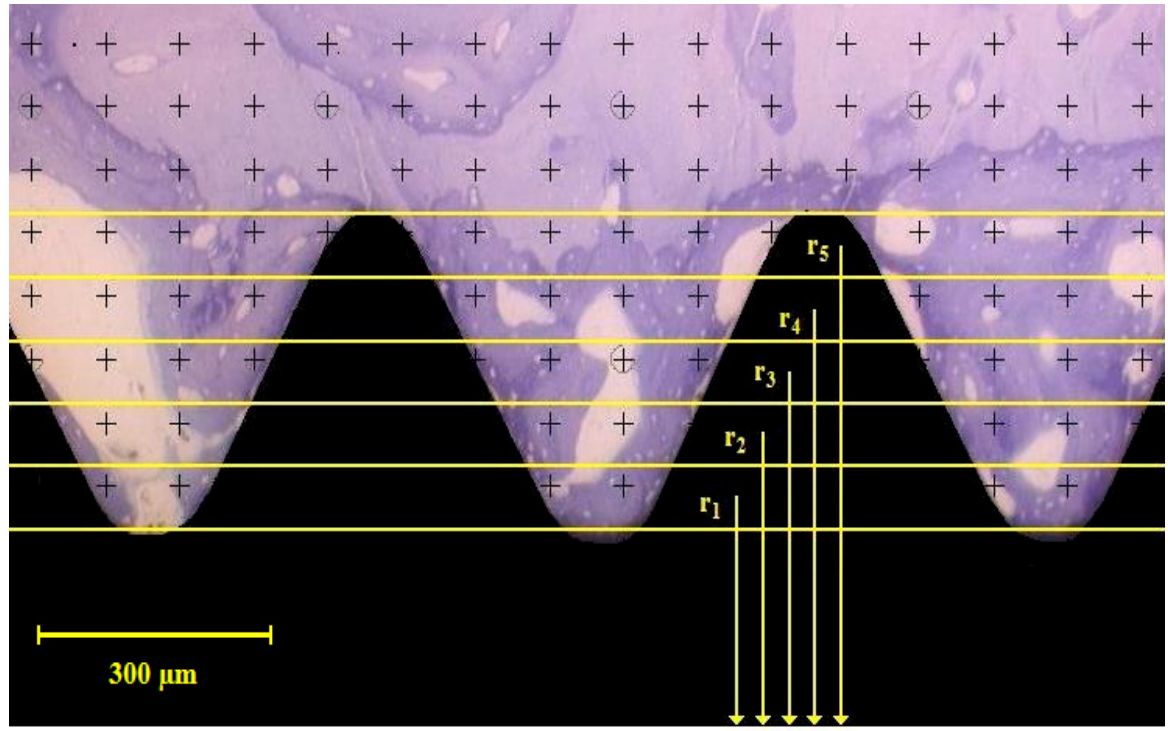

Fig. 7. A-systematic point set was randomly placed over the section, parallel to the axis and for each row of points the distance ( $r$ ) from the row of points to the vertical axis of the implant was measured. The axis of the implant is the lower edge of the image.

\section{VARIANCE AND REPRODUCIBILITY OF THE MEASUREMENTS $\left(\mathrm{S}^{2}\right)$}

The real precision of $B I C$ and $B D-i$ measurements, given the section, was estimated empirically from replicated measurements of all sections in the 3 specimens (Table 2 and 3). As there were no systematic differences between the measurements made on two occasions, the variance due to estimation (Noise), $S^{2}$, given the sections, was calculated using the formula:

$$
S^{2}=\left\lfloor\sum d^{2}\right] / 2 n \text {. }
$$

Where $d$ is the difference between a pair of repeated measurements and $n$ is the number of measured pairs. The $C E$ was calculated as $\mathrm{S} / \mathrm{X}$, where $\mathrm{X}$ is the mean of the measurements.

Table 2. The coefficient of error for the reproducibility (CE $\left.E_{\text {Noise }}\right)$ of Bone-to-Implant Contact (BIC) measurements, the $C E_{\text {syst }}$ between rotated sections (3 bone/implant blocks sectioned into 4 specimens using the FAVER technique). The averaging of $\overline{B I C}$ is over the two replications on the same sections.

\begin{tabular}{|c|c|c|c|c|c|c|c|}
\hline & & & & & $\mathrm{BIC} \%$ & & \\
\hline Animal & Section & $\mathrm{BIC}_{1 \mathrm{st}}$ & $\mathrm{BIC}_{2 \mathrm{nd}}$ & Mean & $\overline{B I C}$ & $C E_{\text {Noise }} \%$ & $C E_{\text {Syst }} \%$ \\
\hline & 1 & 55.9 & 56.5 & 56.2 & & & \\
\hline 1 & 2 & 64.8 & 62.2 & 63.5 & & & \\
\hline 1 & 3 & 55.5 & 55.5 & 55.5 & 58.6 & 2.7 & 5.8 \\
\hline & 4 & 60.9 & 57.4 & 59.2 & & & \\
\hline & 1 & 27.7 & 27.7 & 27.7 & & & \\
\hline 2 & 2 & 58.1 & 59.6 & 58.9 & & & 67 \\
\hline 2 & 3 & 43.3 & 47.2 & 45.3 & 40.8 & 3.6 & 6.2 \\
\hline & 4 & 31.6 & 31.4 & 31.5 & & & \\
\hline & 1 & 8.4 & 15.4 & 11.9 & & & \\
\hline & 2 & 29.9 & 31.9 & 30.9 & & & \\
\hline 3 & 3 & 63.3 & 66.2 & 64.8 & 38.4 & 8.9 & 10.4 \\
\hline & 4 & 43.2 & 48.8 & 46.0 & & & \\
\hline & & Mea & f $3 \mathrm{sp}$ & nens & 45.9 & 5.8 & 7.8 \\
\hline
\end{tabular}




\section{COEFFICIENT OF ERROR BETWEEN SECTIONS (CESYST)}

The average coefficient of error $\left(C E_{\text {Syst }}\right)$ due to the random rotation of the set of the 4 sections can be computed using the following equation (Gundersen et al., 1999):

$$
C E_{s y s t}=\frac{\sqrt{\left[3\left(A-S^{2}\right)-4 B+C\right] / 240+S^{2}}}{\sum_{i=1}^{i=4} f_{i}}
$$

where $S^{2}$ is the variance representing the "Noise" of the measurements, described in the previous section, and mean measurement of the 4 sections are denoted $f_{i}$ and $A=\sum_{i=1}^{n} f_{i}^{2}, B=\sum_{i=1}^{n-1} f_{i} f_{i+1}, C=\sum_{i=1}^{n-2} f_{i} f_{i+2}$. For the estimation of $C E_{S y s t}(B I C), S^{2}$ is the sum of the above Noise and the variance from the test system, shown in Table 1 as a CE.

\section{THE OBSERVED BIOLOGICAL VARIATION (CV)}

The observed biological variation is described by the coefficient of variation ( $\mathrm{CV}=\mathrm{SD} / \mathrm{mean}$ ) among specimens. As the number of animals in the pilot study was limited to only 3 the $C E$ was compared with $C V(B I C)$ and $C V(B D-i)$ from the following experimental study including 16 animals (Balatsouka et al., 2005).

\section{RESULTS}

For $B I C$ measurements done with the unbiased stereological design each intersection line was characterized with a mean weight or contribution $\left(\bar{w}_{i}\right)$ and represented different values depending on the distance from the intersection point $\left(I_{i}\right)$ to the axis of the implant and the angle between the line and the implant surface (Table 1). Thus, lines in the bottom of the implant threads, close to the implant axis, e.g. intersection line 4 , represented a smaller surface area than lines hitting the implant at top of the implant thread. Lines hitting the surface obliquely, e.g., line 2 and 6 , represented a greater surface area than lines hitting the surface more perpendicular, e.g., line 4 and 7 (Table 1). The mean $C E\left(\bar{w}_{i}\right)$ of the test system was 5.5 to $5.7 \%$ (Table 1 ).

The mean $C E_{\text {Noise }}$ of the difference between the 2 sets of repeated estimates of $B I C$ was $5.8 \%$, close to, but above the $C E\left(\bar{w}_{i}\right)$ contributed by the test system itself (Table 2). The mean $C E_{\text {Noise }}$ of the difference between the 2 sets of repeated estimates for $B D-i$ was $7.7 \%$ (Table 3 ). These percentages indicated that both methods turned out to be rather precise.

The biological variation $(\mathrm{CV})$ between the 16 animals in further studies was $19 \%$ for the $B I C$ estimates and $24 \%$ for the $B D-i$ estimates.

For the $B I C$ estimates the mean $C E_{\text {Syst }}$ between the rotated sections was $7.8 \%$ and for the $B D-i$ estimates it was 5.7\% (Tables 2 and 3).

Table 3. The coefficient of error for the reproducibility ( $\left.C E_{\text {Noise }}\right)$ of peri-implant Bone Density (BD) measurements, the $C E_{\text {Syst }}$ between sections rotated (3 bone-implant blocks sectioned into 4 specimens using the FAVER technique). The averaging of $\overline{B D}$ is over the two replications on the same sections.

\begin{tabular}{|c|c|c|c|c|c|c|c|}
\hline \multirow{2}{*}{ Animal } & \multirow[b]{2}{*}{ 1. Section } & \multicolumn{6}{|c|}{$\mathrm{BD} \%$} \\
\hline & & $\mathrm{BD}_{1 \mathrm{st}}$ & $\mathrm{BD}_{2 \mathrm{nd}}$ & Mean & $\overline{B D}$ & $C E_{\text {Noise }} \%$ & $C E_{\text {Syst }} \%$ \\
\hline \multirow{4}{*}{1} & 1 & 49.8 & 57.7 & 53.8 & \multirow{4}{*}{52.3} & \multirow{4}{*}{7.1} & \multirow{4}{*}{7.0} \\
\hline & 2 & 55.7 & 61.0 & 58.4 & & & \\
\hline & 3 & 37.5 & 40.6 & 39.1 & & & \\
\hline & 4 & 56.5 & 59.8 & 58.2 & & & \\
\hline \multirow{4}{*}{2} & 1 & 18.9 & 25.1 & 22.0 & \multirow{4}{*}{27.8} & \multirow{4}{*}{9.4} & \multirow{4}{*}{6.7} \\
\hline & 2 & 33.5 & 37.0 & 35.3 & & & \\
\hline & 3 & 34.7 & 34.4 & 34.6 & & & \\
\hline & 4 & 19.2 & 19.7 & 19.5 & & & \\
\hline \multirow{4}{*}{3} & 1 & 13.6 & 12.7 & 13.2 & \multirow{4}{*}{24.5} & \multirow{4}{*}{6.5} & \multirow{4}{*}{2.1} \\
\hline & 2 & 26.3 & 26.6 & 26.5 & & & \\
\hline & 3 & 35.4 & 33.9 & 34.7 & & & \\
\hline & 4 & 21.5 & 25.7 & 23.6 & & & \\
\hline \multicolumn{5}{|c|}{ Mean of 3 specimens } & 34.9 & 7.7 & 5.7 \\
\hline
\end{tabular}




\section{DISCUSSION}

The stereological design used in the present study is the optimal way to obtain unbiased estimates of $B I C$ and $B D-i$ on bone-implant sections. The technique makes it possible to obtain unbiased and efficient estimates of $B I C$ and $B D-i$ from 4 randomly and systematically sampled $2 \mathrm{D}$ sections. The good efficiency of the coaxial sections stems from two effects in combination: systematic sampling of directions and systematic sampling of locations, by which the 4 sections explore the whole sampling universe around the implant in a favourable manner.

The mean $C E\left(\bar{w}_{i}\right)$ represents the efficiency of our method. The low mean $C E\left(\bar{w}_{i}\right)$ values demonstrate that our method is very efficient (Gundersen et al., 1999). Compared to the biological variation between animals $(C V)$, the $C E\left(\bar{w}_{i}\right)$ indicates that the sampling and estimation design is efficient and does only have a small effect on the variation of the final $B I C$ and $B D-i$ values.

For unbiased estimation of bone to implant contact length $(B I C)$ at the surface of screw-formed implants, intersections close to the implant axis represent smaller surface areas than more peripheral intersections and intersections hitting an oblique part of the screw implant represent a greater surface area than intersections hitting the implant perpendicular to the implant axis. For unbiased estimation of the bone density $(B D-i)$ in the bone threads the points close to the implant surface (axis) represent a smaller volume than points in more peripheral locations. Thus, in the present study intersections hitting the surface at oblique parts close to the outer part of the thread represented a 2 to 3 times greater surface area than intersections hitting the surface perpendicular and in the bottom of the implant threads. This is in accordance with a three-dimensional evaluation and in contrast to a two-dimensional evaluation, where each line and point is given the same weight.

Careful planning of the sampling method before the specimens and sections are processed is mandatory for obtaining unbiased estimates of bone implant variables in research on bone implants (Overgaard et al., 2000). In the present study, substantial variations in $B I C$ and $B D-i$ were demonstrated between 4 sections from the same bone-implant block. This demonstrated that the values for $B I C$ and $B D-i$ may be quite dependent on the direction in which the bone/implant blocks are sectioned, which also have been indicated in histomorphometric studies using two-dimensional analysis (Gotfredsen et al., 1991). This variance included variance from the counting procedure as well as true variance from section to section (Vesterby et al., 1987). In the present study the variation between rotated $B I C$ sections contributed a $C E_{S y s t}$ of $7.8 \%$. The variation between rotated $B D-i$ sections contributed a $C E_{S y s t}$ of $5.7 \%$. The biological variation for the $\mathrm{BIC}$ and the $\mathrm{BD}$ measurements found in the following experimental study (Balatsouka et al. 2005a) indicated that the precision of our method is very good and $\mathrm{CE}_{\text {Syst }}^{2}$ was less than a fifth of $\mathrm{CV}^{2}$ for both measurements. The moderate impact of the rather pronounced variation from section to section in the four sections is due to systematic sampling, which is superior to ordinary (independent) sampling (Gundersen et al. 1999). The 'price' one has to pay is that the estimation of the precision in systematic designs is much more complicated.

Applying the three dimensional vertical section method on anisotropic bone specimens, unbiased estimates of surface areas have been achieved (Baddeley et al., 1986; Vesterby et al., 1987). When bone-implant interfaces have to be evaluated on rotational symmetric implants the basic requirements for the vertical section method should be followed (Overgaard, 2000). When exhaustively cut serial sections of bone-implant specimens are produced a "shadow effect" will, however, occur at the peripheral sections (Johansson, 1991; Rahbek, 2002). To obtain uniformly, random and systematic cuts through a bone-implant block without any "shadow effect" the "revolving door" or fixed vertical random sectioning technique, described in the present paper, can be used. By this technique the specimen is rotated randomly around a vertical axis, which is defined as the long axis of the implant and cutting was performed after systematically rotating the specimen $90^{\circ}$. However, the sectioning technique was a technical challenge, where X-rays before cutting and re-embeddings were necessary. Thus, the technique was not only more difficult but also more time consuming and expensive compared to the serially cutting technique with a random rotation (ordinary vertical sections) (Gotfredsen et al., 1993).

An optimal sampling design should be based on enough sections rather than measure too few more precisely (Gundersen and Osterby, 1981). However, if the sampling design is efficient the number of sections for analysis could be reduced to four per implant without significantly increasing the variance (Overgaard, 2000; Overgaard et al., 2000). It has been demonstrated, that using systematic random sampling on all levels, quantification can be performed 
by counting 100 to 200 points or intersections on 4 to 10 sections with an acceptable coefficient of error (Gundersen \& Jensen 1987).

\section{ACKNOWLEDGEMENTS}

The study was supported with grants from the ITI Foundation for the Promotion of Oral Implantology, Switzerland and the FUT and Calcin Foundation (Danish Dental Association). We are grateful to Karen Biré for assistance in sectioning. We are also grateful to $\mathrm{Mr}$ George Anthis for his help in measuring the angles. We also want to acknowledge the help of the associate professor Jytte Overgaard (Department of Medical Anatomy, University of Copenhagen) for the use of her microscope for our stereological measurements.

\section{REFERENCES}

Albrektsson T, Brånemark PI, Hansson H, Lindström J (1981). Osseointegrated titanium implants. Requirements for ensuring a long-lasting, direct bone-to-implant anchorage in man. Acta Orthopaedica Scandinavica 52:155-70.

Albrektsson T (1988). A multicenter report on osseointegrated oral implants. J Prosthet Dent 60:75-84.

Baddeley AJ, Gundersen HJG, Cruz-Orive LM (1986). Estimation of surface area from vertical sections. J Microsc 142:259-76.

Balatsouka D, Gotfredsen K, Lindh $\mathrm{CH}$, Berglundh T (2005). The impact of nicotine on bone healing and osseointegration. An experimental study in rabbits. Clin Oral Impl Res 16:268-76.

Cruz-Orive LM (1987). Stereology: recent solutions to old problems and a glimpse into the future. Proc. ICS VII Caen 1987. Acta Stereol 6(III):3-18.

Cruz-Orive LM, Weibel ER (1990). Recent stereological methods for cell biology: a brief survey. A J Physiol 258:L148-56.

Cruz-Orive LM, Roberts N (1993). Unbiased volume estimation with coaxial sections: an application to the human bladder. J Microsc 170:25-33.

Fini M, Gianluca G, Rimondini L, Giardino R (2002). Titanium Alloy Osseointegration in Cancellous and Cortical Bone of Ovariectomized Animals: Histomorphometric and Bone Hardness Measurements. Int J Oral Maxillofac Implants 17:28-37.

Gotfredsen K, Budtz-Jorgensen E, Jensen LN (1989). A method for preparing and staining histological sections containing titanium implants for light microscopy. Stain Technol 64:121-7.

Gotfredsen K, Rostrup E, Hjörting-Hansen E, Stoltze K, Jörgensen B (1991). Histological and histomorphometric evaluation of tissue reactions adjacent to endosteal implants in monkeys. Clin Oral Impl Res 2:30-7.
Gotfredsen K, Nimb L, Buser D, Hjørting-Hansen E (1993). Evaluation of guided bone regeneration around implants placed into fresh extraction sockets. An experimental study in dogs. Int J Oral Maxillofac Surg 51:879-84.

Gotfredsen K, Berghlund T, Lindhe J (2000). The anchorage of titanium implants with different surface characteristics. An experimental study in rabbits. Clin Impl Dent Rel Res 2:120-8.

Gotfredsen K, Berglundh, Lindhe J (2001). Bone reactions adjacent to titanium implants subjected to static load. A study in the dog (I). Clin Oral Impl Res 12:1-8.

Gundersen HJG, Osterby R (1981). Optimizing sampling efficiency of stereological studies in biology: or 'do more less well'. J Microsc 121:65-73.

Gundersen HJ, Bendtsen TF, Korbo L, Marcussen N, Møller A, Nielsen K et al. (1988). Some new, simple and efficient stereologics methods and their use in pathological research and diagnosis. APMIS 96:379-94.

Gundersen HJG, Jensen EB (1987). The efficiency of systematic sampling in stereology and its prediction. $\mathrm{J}$ Microsc 147:229-63.

Gundersen HJG, Jensen EB, Kieu K, Nielsen J (1999). The efficiency of systematic sampling in stereologyreconsidered. J Microsc 193:199-211.

Ivanoff CJ, Sennerby L, Johansson C, Rangert B, Lekholm $U$ (1997). Influence of implant diameters on the integration of screw implants. An experimental study in rabbits. Int J Oral Maxillofac Surg 26:141-8.

Jensen EBV, Gundersen HJG (1993). The rotator. J Microsc 170:35-44.

Johansson CB (1991). On tissue reactions to metal implants. PhD dissertation, Götenborg, Sweden, ISBN: 91-628-0223-2.

Kolonidis S, Renvert S, Hämmerle CHF, Lang N, Harris D, Claffey N (2003). Osseointegraion on implant surfaces previously conamnated with plaque. An experimental study in the dog. Clin Oral Impl Res 14:373-80.

Miranov JrAA, Miranov AA (1998). Estimation of subcellular organelle volume from ultra-thin sections through centrioles with a discretized version of the vertical rotator. J Microsc 192:29-36.

Overgaard S, Bromose U, Lind M, Bunger C, Soballe K (1999). The influence of crystallinity of the hydroxyapatite coating on the fixation of implants. Mechanical and histomorphometric results. J Bone Joint Surgery Br 81:725-31.

Overgaard S (2000). Calcium phosphate coatings for fixation of bone implants evaluated mechanically and histologically by stereological methods. $\mathrm{PhD}$ dissertation. Acta Orthopaedica Scandinavica 71 (Suppl 297):1-74.

Overgaard S, Soballe K, Gundersen HJG (2000). Efficiency of systematic sampling in histomorhometric bone research illustrated by hydroxyapatite-coated 
implants: Optimizing the stereological vertical-section design. J Orthop Res 18:313-21.

Rahbek O (2002). The influence of hydroxyapatite coating on the peri-implant migration of polyethylene particles. $\mathrm{PhD}$ dissertation, Aarhus, Denmark.

Rasmusson L, Kahnberg KE, Tan A (2001). Effects of Implant Design and Surface on Bone Regeneration and Implant Stability: An Experimental Study in the Dog Mandible. Clin Impl Dent and Rel Res 3:2-8.

Romanos GE, Toh CG, Siar CH, Swaminathan D (2002). Histologic and Histomorphometric Evaluation of Periimplant Bone Subjected to Immediate Loading: An
Experimental Study with Macaca Fascicularis. Int J Oral Maxillofac Implants 17:44-51.

Schou S, Holmstrup P, Skovgaard LT, Hjørting-Hansen E, Gundersen HJG (2003). Autogenous bone graft and ePTFE membrane in the treatment of peri-implantitis. II. Stereologic and histologic observations in cynomolgus monkeys. Clin Oral Impl Res 14:404-11.

Sennerby L, Ericson LE, Thomsen P, Lekholm U, Åstrand P (1991). Structure of the bone-titanium interface in retrieved clinical oral implants. Clin Oral Impl Res 2:103-11.

Vesterby A, Kragstrup J, Gundersen HJG, Melsen F (1987). Unbiased stereologic estimation of surface density in bone using vertical sections. Bone 8:13-7. 